\title{
Jeremy Wright \\ Innovation in minimal access surgery: changing the culture?
}

Received: 20 December 2004/ Accepted: 15 March 2005/Published online: 21 May 2005

(C) Springer-Verlag Berlin / Heidelberg 2005

Currently we are being exposed in the UK to what is known as "the Nanny State". I have recently endured an afternoon playing with dolls to ensure that my resuscitation skills are up-to-date if I am called to a cardiac arrest or a choking child, and happily I have been awarded two certificates for this. Next week, a consultant microbiologist is attending to ensure that I know how to wash my hands properly in an effort to beat new killer bugs.

In general terms, this is all very well, but in the keyhole world of minimal access surgery, "Nanny" turns to "Stalin" with a committee of cultural correctness hovering over colleagues who wish to implement an innovative approach to the management of their patients. Relatively newly appointed consultants who arrive enthused with new ideas and new training are blocked or criticised by their older peers, for example, for undertaking a laparoscopic hysterectomy when "this could easily be achieved through a small Pfannenstiel". A complication, such as vault haematoma, perfectly acceptable at open surgery, becomes a major complication because the surgery has been performed using minimal access techniques, and becomes the subject of incident inquiries. Dossiers are kept, the young surgeon becomes more paranoid and anxious, he/she becomes extremely stressed and frustrated, and stops teaching trainees for fear that something may go wrong. This is even more greatly magnified in the field of endometriosis surgery, where the evidence base for surgical treatment is poor and the evidence base for medical treatment stronger, the latter pointing to its general ineffectiveness. The increasing evidence base, although purely that of case series, is that surgery for severe recto-vaginal endometriosis, albeit often requiring two or more surgical episodes, is effective and, in the vast majority of cases, safe. However, like open surgery on the bowel,

\section{J. Wright}

Woking Nuffield Hospital, Shores Road, Woking,

Surrey, GU21 4BY, United Kingdom

E-mail: jwrighta@cix.co.uk faecal leakage can occur and can be life-threatening, or result in a recto-vaginal fistula requiring extensive surgery to facilitate a repair. The risks are, however, no different from those arising from anterior resection performed at open surgery, but unlike anterior resection successful laparoscopic surgery involves only a few days in hospital and a much more rapid recovery.

Laparoscopic surgery allows repeat laparoscopy and adhesiolysis, while anterior resection through a midline incision does not. Because of cross-specialisation, however, unless there is a good rapport with an appropriately trained colorectal surgeon (and these are thin on the ground), complications following such surgery (which is usually long and tedious itself) will inevitably result in whispered innuendo and mutterings of "incompetence" and "cowboy-like behaviour" by the surgeon, followed by an "independent" inquiry and censure by the culturally correct.

How then, does the innovative surgeon break free from the Kuhnian paradigm? This, in gynaecology, refers to the safety of laparotomy and of third generation endometrial ablation techniques which, although having the advantages of being skill-free and increasingly safe, have the drawback of ineffectiveness in $70 \%$ of cases. The great difficulty, then, of breaking through the barrier, that we all recognise from applying for a research grant, is that science is increasingly being planned and we are being invited to research on areas of government interest, and that areas that are new and innovative are brushed away as absurd and fanciful. A good historical example is the Wertheim/Schauta debate in the nineteenth century. Wertheim had a better operation than Schauta initially, that allowed for gland dissection. However, the initial mortality rate was so high that Schauta's less radical procedure was introduced. Having witnessed one of these in Austria, the Oberarzt remarked to me afterwards, "the thing about this operation was that the patient would remember it every time she sat down". With improved anaesthesia and the availability of blood, the Schauta procedure was airbrushed out of history in an almost Orwellian way, but it is interesting 
that now, with a paradigm shift and considerable distrust from those undertaking Wertheim's procedure, the Schauta procedure is being reintroduced by those oncologists trained in laparoscopic surgery and able to dissect pelvic glands, in some cases with the entrenched opposition of those trained in more conventional oncology.

The appropriate view is of course the Popperian one, in which new procedures should be welcomed but subject to test and audit and accepted if they are seen to be successful and changed if not. Sadly, the Popperian view is almost totally subsumed and this paradigm shift is very difficult to achieve in the current culture of political correctness.

For a fascinating discussion of these areas, you could do worse than read Kuhn vs Popper: the Struggle for the Soul of Science by Steve Fuller (Columbia University Press, ISBN1-84046-468-2).

How then do we protect doctors from their peers, if these doctors believe that the innovative surgery they have to offer-be it achieving vaginally what others can only offer by open abdominal surgery, or by laparoscopic treatment what others can only suppress with unpleasant medication - can help their patients?

Clinical trials might seem the answer, but leaving aside the enormous logistic and intellectual difficulties of undertaking a good clinical trial for a surgical procedure, the regulation of clinical trials and the ill informed nature of ethical committees with their equally distorted Kuhnian paradigms makes such ventures likely to fall at the first post.

The answer currently lies in meticulous audit and case series. These are presently the only way of protecting the individual and at least accurately assessing the effectiveness and pitfalls of innovative surgery. If those critical of this surgery were to audit the results from their own open surgery they would find their own techniques to be wanting too.

Certainly the large audit of surgical practice in such operations as hysterectomy carried out in areas such as
Finland, where it is possible to collect the data, suggest that morbidity is considerably greater than most of us believe of our own practice.

Those who wish to try innovative things to help their patients will, I'm afraid, have to continue in the teeth of opposition from their generalist peers. They will have the respect of their patients provided they are properly informed. These patients are usually desperate to have something approaching a normal life, having had numerous failed treatments before, so it is important that their expectations are not allowed to be unreal, however difficult this may be.

Patient power however is very limited in the Nanny State; the Stalinist forces of the committee of cultural correctness present in most hospitals pay mere lip service to what our patients want. They know best.

The continental model is in fact no better. The hierarchical nature of the university hospital means that only enlightened department heads will allow innovative surgery unless there is financial advantage. Most hospitals serve small communities and any complication is likely to reflect badly on a hospital, so it is either covered up or treatment is inappropriate to avoid the possibility of complication. Patients in Europe are now voting with their feet, travelling several hundreds or thousands of miles to seek the treatment they need from specialist centres. These cases go mostly unreported because of the small numbers involved. Protection for surgeons must therefore come from working in centres with groups committed to undertaking the surgery with the appropriate audit and feedback. I fear that working in isolation in small units will only lead to criticism and difficulty. The question is, of course, whether we will be able to serve our patients in these units, or whether the natural conservatism of our peers will continue to prevent us from trying to develop the best possible treatment for those patients with intractable conditions.

The future at the moment is bleak, but with proper training, audits, and the establishment of centres of excellence, it could actually change. 